Article

\title{
Facilities Design Based on Apparent Motion of Grating for Speed Reduction in Tunnel
}

\section{Bing Liu *, Shunying Zhu, Hong Wang and Jing Xia}

School of Transportation, Wuhan University of Technology, Wuhan 430063, China; E-Mails: zhusy2001@163.com (S.Z.); wanghong2004317@sina.com (H.W.); xiajingjt@126.com (J.X.)

* Author to whom correspondence should be addressed; E-Mail: xiajingtangba@whut.edu.cn; Tel./Fax: +86-27-8655-1193.

Academic Editor: Marc A. Rosen

Received: 23 September 2014 / Accepted: 20 January 2015 / Published: 30 January 2015

\begin{abstract}
This paper introduces the concept of apparent motion, and by examining the characteristics of apparent motion and of velocity vectors, puts forward a new speed reduction method in tunnels. Finally, we verify this effect through a simulation experiment. The experiment first used 3DMAX to make simulation videos with apparent motion grating installed on the tunnel wall, and then took the "stable same, direction movement" proportion of perception responses as the index to determine the optimal form of apparent motion. Using the observations of six males and two females, the experiments show that, with a space layout of two bright, two dark or four bright, four dark, a stimulus separation (SS) of 2 or $4 \mathrm{~m}$, and stimulus onset asynchrony (SOA) of $60,120,180$ or $240 \mathrm{~ms}$, participants tended to perceive apparent motion as "stable same, direction movement". Based on the above, 16 combinations of grating were adopted as optimal forms. By using the Forced-Choice Method, the experiments showed that the best parameters of apparent motion grating for speed reduction are: two bright, two dark, SS as $4 \mathrm{~m}$ and SOA as $60 \mathrm{~ms}$. Under these conditions, the average perceived speed of eight observers reaches the maximum; meanwhile, the standard deviation is lower than that of the four dark, four bright case.
\end{abstract}

Keywords: tunnel; apparent motion; speed perception; speed reduction; edge rate 


\section{Introduction}

If an accident does happen in a tunnel, the severity of injuries sustained is significantly higher than on open stretches of motorways. According to traffic accident statistics, more than $60 \%$ of accidents in tunnels are due to the driver speeding [1]. Now, the main measures for driving speed reduction in tunnels are vibration deceleration zones, speeding snapshots and the speed limit sign, but these methods all have their defects. Vibration deceleration stems from sensations felt by the driver, whereby the vibration causes the driver to feel uncomfortable at a high speed so the speed is reduced. However, the slowing effect of vehicle vibration reduction may be reduced with improvements in vehicle performance. In addition, vibration will also cause some damage to vehicles. The speeding snapshot system is a method that punishes and deters the driver after a speeding occurrence, which may not prevent an accident occurring in the first place. The speed limit sign also does not have a strong slow-down effect because drivers often ignore the signs. A new theory and method for vehicle speed forced control is urgently needed. Estimations suggest that over $90 \%$ of all traffic accidents are related to human error, which indicates that it is important to adjust road design according to the limitations of human information processing [2]. Thus, a method for forced control should be based on comprehensive coordination of people-car-road-environment factors. More importantly, it must stem from the most significant aspect of speed control - the determinants of a driver's perception of speed.

In view of this, apparent motion [3] and velocity vector characteristics, from the driver's perspective, are studied using a speed control method in a tunnel based on apparent motion of grating.

\section{Design Theory}

\subsection{Speed Control Process}

Recarte [4] studied the relationship between physical speed and perceived speed, who found that with a decrease of perceived speed, the physical speed may increase. The ratio of perceived speed and physical speed increments is about 2:1; conversely, the increase of perceived speed can also lead to the decrease of actual speed. For that reason, this study plans to reduce speed through the change of the driver's perceived speed using the speed reduction process shown in Figure 1.

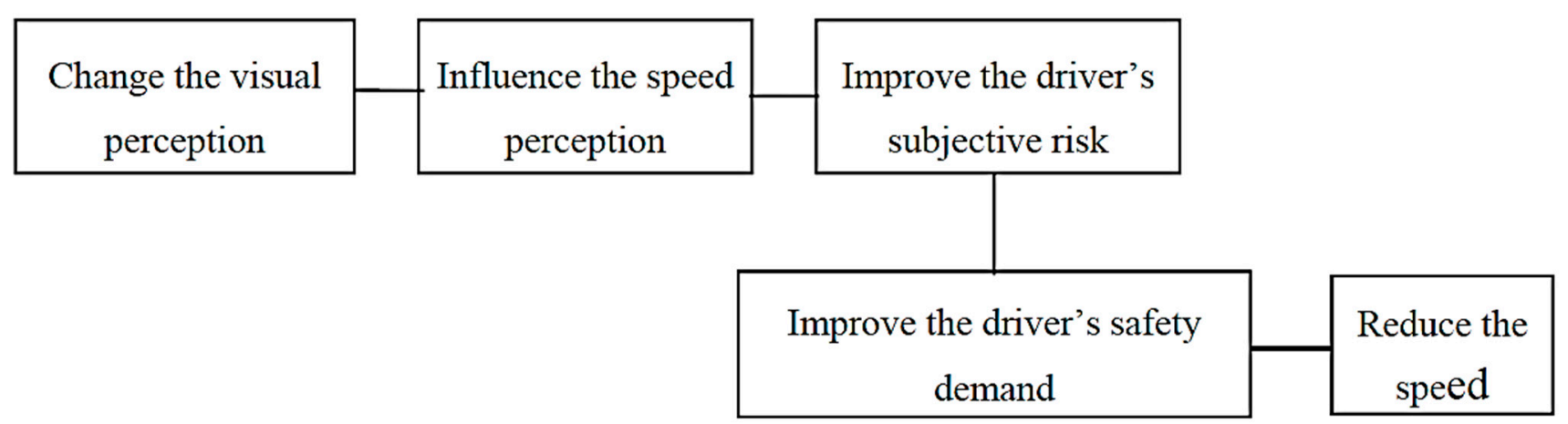

Figure 1. Process of speed reduction based on speed perception. 


\subsection{Apparent Motion}

In the early 20th century, German psychologist Max Wertheimer used experimental methods to research the phenomenon of apparent motion [5]. He pointed out that if two stimuli are briefly flashed in rapid succession, with appropriate spatial and temporal displacements, observers will report seeing an illusory motion between the two stimuli. This perceptual phenomenon is called apparent motion. SOA (stimulus onset asynchrony) and SS (stimulus separation) are the main phenomena to influence the effect of apparent motion. He successively made two bright line segments ( $a, b)$, changed the presented SOA of the two line segments, and measured their perceptual experience. It was found that when two line segments of the SOA are shorter than $30 \mathrm{~ms}$, people see $\mathrm{a}$ and $\mathrm{b}$ at the same time; when the SOA are longer than $200 \mathrm{~ms}$, people see $\mathrm{a}$ and $\mathrm{b}$ as having successively appeared; when the SOA are about $60 \mathrm{~ms}$ apart, people see the movement of the line segment from a to $b$, at which the stimulus intensity (the SOA) is inversely proportional to spatial distance. After that, some other researchers [6-8] conducted similar studies, and they came to the same conclusion. When setting up the apparent motion grating in the tunnel wall, if its moving direction is opposite to the vehicle forward direction, according to the velocity vector characteristics, the relative speed between driver and grating should increase. However, these studies used stimulus that appeared in the participants' central vision; and there was a lack of stimulus that appeared in the participants' peripheral vision. When the apparent motion grating is set up on the wall in the tunnel, the apparent motion occurs in the driver's peripheral vision. Cases whereby the apparent motion occurred in the driver's peripheral vision are used by only a few scholars: Tsuda [9] studied the relationship between visual angle and optimal SS of the apparent motion, when the apparent motion occurred in the peripheral vision. Accordingly, the optimal SS of the apparent motion increases with the visual angle's increasing between stimulus and the participants. However, the stimulus apparent motion is side-to-side movement relative to the participants in the above study, lacking back-to-forth movement. As the velocity is vector-based, when the moving direction of apparent motion grating in the tunnel is opposite to the vehicle's forward direction, this may easily lead the driver to overestimate the driving speed. Through the above analysis of Wertheimer's and Tsuda's studies, we chose a different experimental environment for this study to all previous studies, in order to examine the best form of apparent motion.

\section{Experimental Section}

\subsection{Experiment 1: Optimal Form of Apparent Motion}

When using the apparent motion of grating for speed control, attention should be paid to two questions: First, improper control of the grating SOA and SS will make the observer experience a "flash" feeling; then the grating will lack a sense of movement. Second, apparent motion also produces motion after effect (MAE) [10]. The so-called MAE, also known as the waterfall effect, refers to the following phenomenon: If you look at the waterfall for some time, and then move your fixation point to the adjacent rock rapidly, you will see the rock moving up. If the apparent motion grating is conducive to creating an MAE for the driver, the driver will not be able to obtain a stable speed perception. The apparent motion grating for speed control should work to avoid the above two phenomena. The optimal apparent motion 
grating should make the driver obtain a "stable, same direction movement" perception. Then, the research looked at the optimal form of apparent motion.

\subsubsection{Measurement of Optimal Form of Apparent Motion}

The experiment takes the "stable, same direction movement" proportion as the index to determine the best form of apparent motion.

\subsubsection{Apparatus}

We used HP XW6400 computer graphic work station to generate and control the driving simulation which was projected on a $2.2 \mathrm{~m} \times 2 \mathrm{~m}$ screen using TOSHIBA projector. The selected subjects were seated $1.5 \mathrm{~m}$ away from the screen and the vertical distance between the subject's eyes and screen bottom was $1.2 \mathrm{~m}$.

\subsubsection{Participants}

There were eight subjects who participated in this experiment (six males and two females) and five of them (four males and one female) hold valid driving licenses. They were naïve as to the purposes of the experiment; the eyesight or corrected eyesight of them is no less than 1.0.

\subsubsection{Procedures}

Each participant was shown the stimulus for $3 \mathrm{~min}$, and then they reported orally either: (1) stable, same direction movement; (2) unstable movement (movement in uncertain directions); (3) flashing (only changes between light and shade). The participants experienced all conditions in various, random orders. Each participant repeated the experiment 10 times. After that, we obtained the number of "stable, same direction movement" choices.

\subsubsection{Experiment Scenarios}

The stimuli to be judged goodness of apparent movement were varied in both SS $(2,4,6$, and $8 \mathrm{~m})$ and SOA $(60,120,180$, and $240 \mathrm{~ms})$. There are three layouts for grating: one bright one dark, two bright two dark and four bright four dark. In total, there were $48(4 \times 4 \times 3)$ scenarios for the SSES experiment. The width of each grating is $0.3 \mathrm{~m}$, and length is $1.5 \mathrm{~m}$. 3DMAX was used to simulate experiment scenarios, which are shown in Figure 2. For each scenario, there were eight subjects who participated and each subject was exposed to the experiment 10 times. Additionally, the intended direction of apparent motion was opposite to the direction of vehicle motion. 


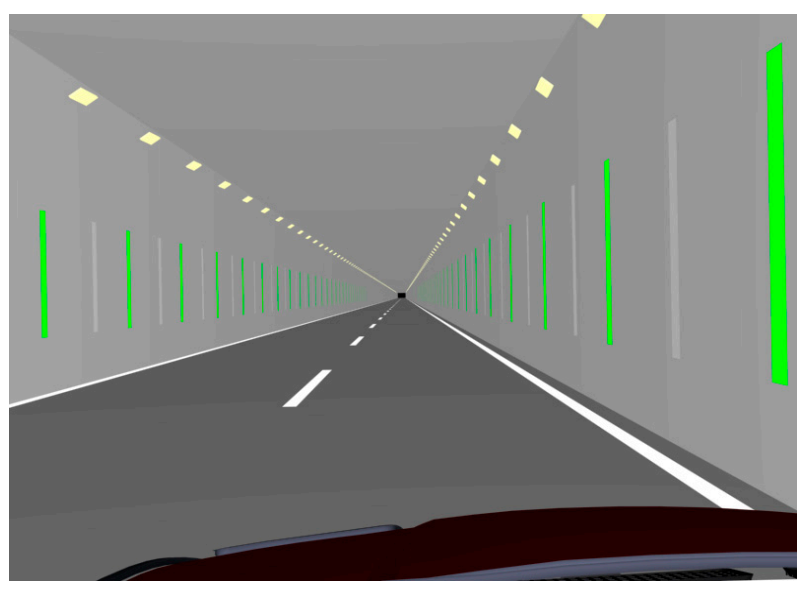

(a)

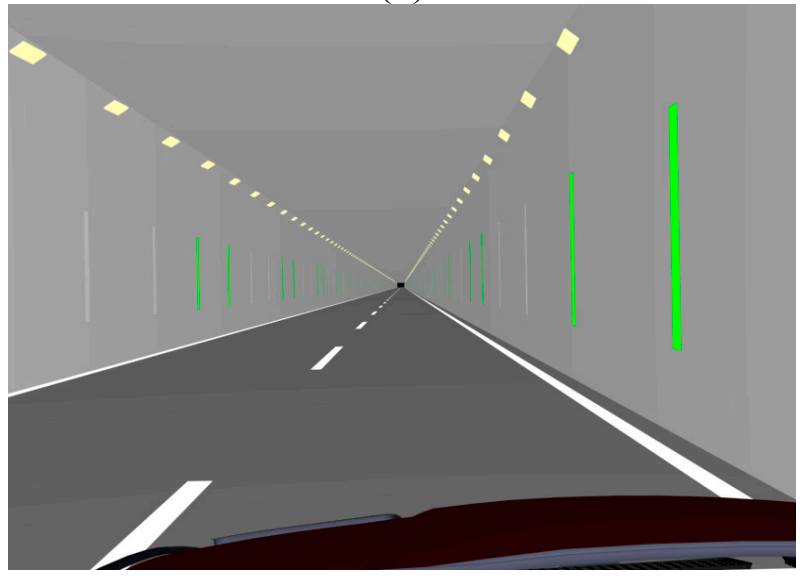

(b)

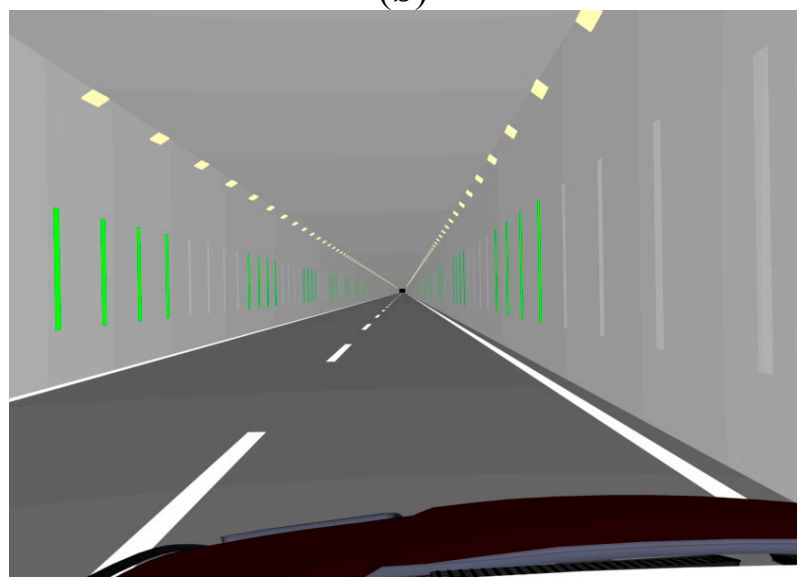

(c)

Figure 2. Experiment scenarios. (a) one bright, one dark; (b) two bright, two dark; (c) four bright, four dark.

\subsubsection{Results}

From a summary of the eight participants' experimental results, we can get the proportion of "stable same, direction movement" under different circumstances, as shown in Figure 3. 


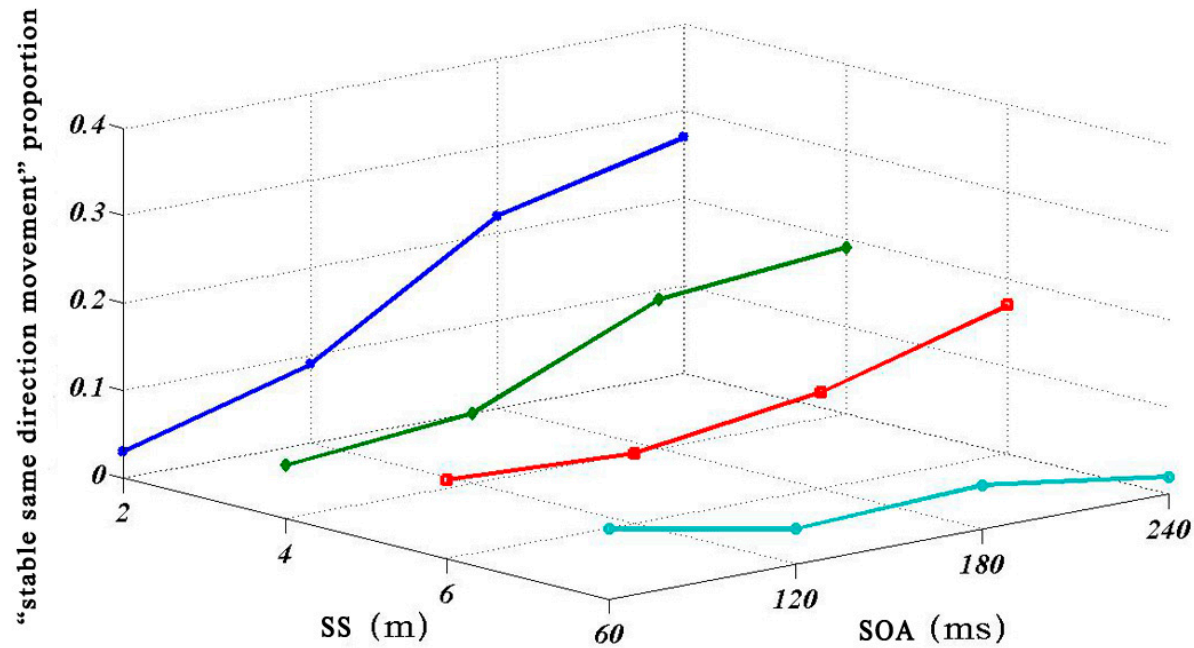

(a)

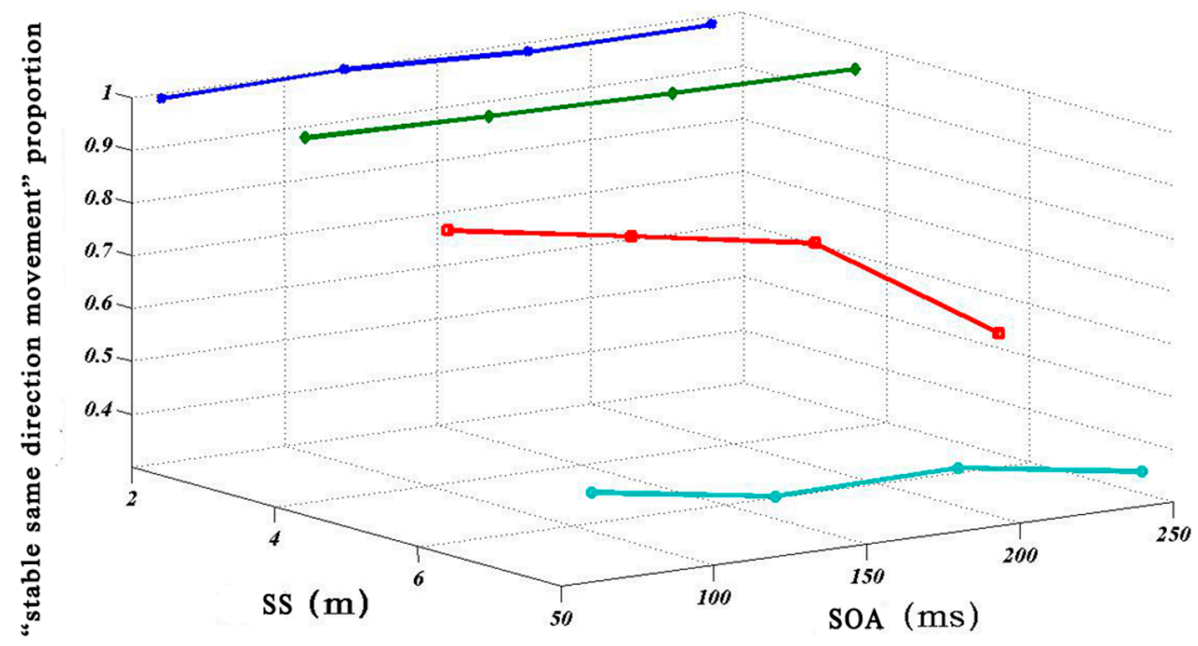

(b)

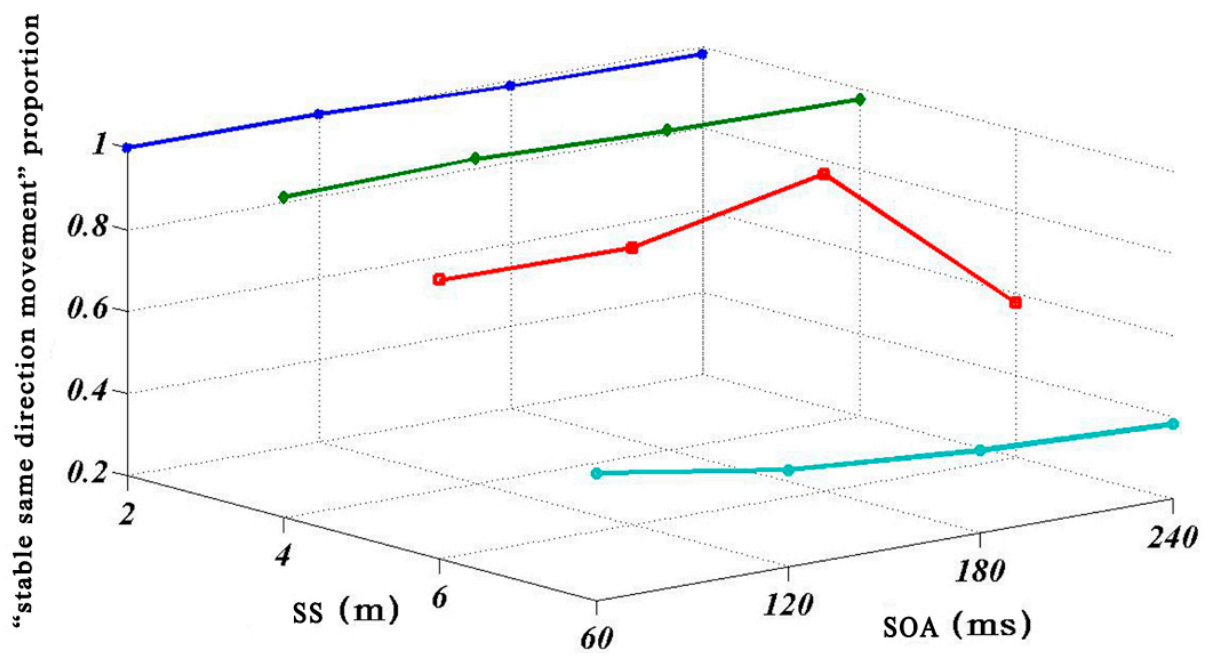

(c)

Figure 3. Results of apparent motion perception. (a) one bright, one dark; (b) two bright, two dark; (c) four bright, four dark. 
From the experimental results, we get the following finding:

(1) When one bright, one dark grating was used, no matter the value of SS and the SOA, participants tended to perceived grating as "flashing" or "unstable movement".

(2) When two bright, two dark or four bright, four dark was used, with the changes of SOA and SS, participants' perceptions of grating changed also. When the SS was $2 \mathrm{~m}$ or $4 \mathrm{~m}$ and the SOA was $60 \mathrm{~ms}, 120 \mathrm{~ms}, 180 \mathrm{~ms}$ or $240 \mathrm{~ms}$, participants tended to perceive grating as "stable, same direction movement". This constituted more than $95 \%$ of perceptions, which differs from the Waite Alzheimer's experimental results. When the SS was $6 \mathrm{~m}$ or $8 \mathrm{~m}$, the proportions reduce gradually. SS and SOA all have significant influence on apparent motion perception; the mean difference was significant at the 0.05 level using Analysis of Variance (ANOVA).

According to the experimental results, the parameters of optimal form of apparent motion grating for speed reduction are listed as follows:

(1) Layout: two bright, two dark, four bright, four dark.

(2) $\mathrm{SS}: 2 \mathrm{~m}, 4 \mathrm{~m}$.

(3) SOA: $60 \mathrm{~ms}, 120 \mathrm{~ms}, 180 \mathrm{~ms}$ and $240 \mathrm{~ms}$.

\subsection{Experiments 2 : Measurement of Driver's Speed Perception}

The parameters of optimal forms of apparent motion were selected according to experiment 1 . Then, the driver's perceived speed is measured to select the best form of apparent motion grating for velocity control.

\subsubsection{Index of the Driver's Perceived Speed}

The experiment adopted Stimulation of Subjectively Equal Speed [11] (SSES) as the performance index to measure the speed that the subject perceives. SSES is the physical speed of "comparison stimuli" when the perceived speed of "comparison stimuli" is equal to the one of "standard stimuli".

\subsubsection{Apparatus and Participants}

The apparatus and participants are the same as in the setup of experiment 1.

\subsubsection{Procedures}

Forced-Choice Method [12] is selected to measure SSES. 


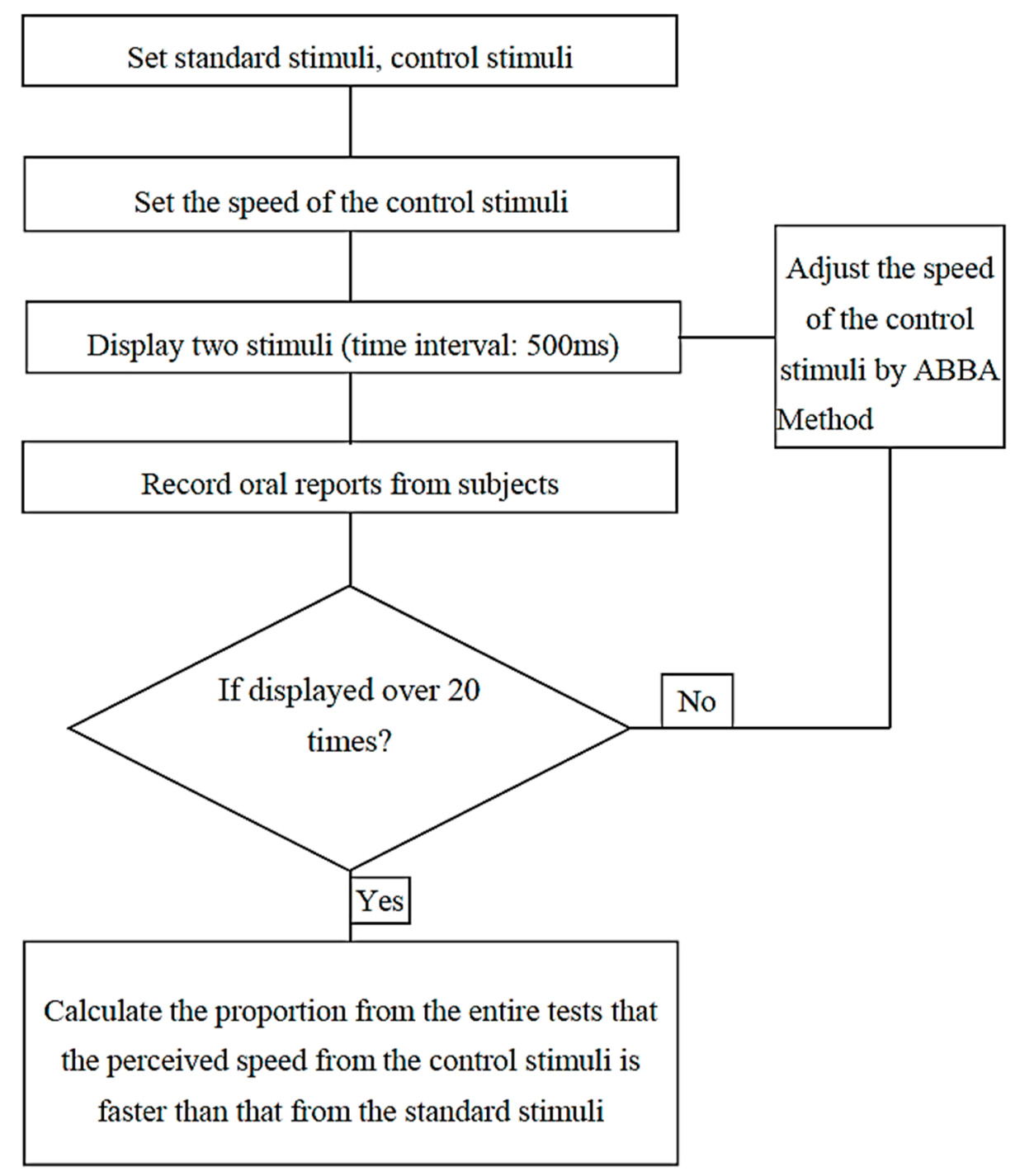

Figure 4. Process of the speed-comparison experiment.

This experiment simulated a series of driving scenarios of different speeds $(60 \mathrm{~km} / \mathrm{h}, 90 \mathrm{~km} / \mathrm{h}, 120 \mathrm{~km} / \mathrm{h})$ using two designs (two bright, two dark, and four bright, four dark), and SS chosen were $2 \mathrm{~m}$ and $4 \mathrm{~m}$, and SOA chosen were $60 \mathrm{~ms}, 120 \mathrm{~ms}, 180 \mathrm{~ms}$, and $240 \mathrm{~ms}$. These simulations were used as standard stimuli. The experiment also simulated a series of driving scenarios which without grating of different speeds (from $40 \mathrm{~km} / \mathrm{h}-200 \mathrm{~km} / \mathrm{h}$, with step-rate $10 \mathrm{~km} / \mathrm{h}$ ), and these simulations were used as comparison stimuli. Participants were instructed to judge the speed they perceived from both scenarios of the standard stimuli and the control stimuli.

In total, there were $816(3 \times 2 \times 2 \times 4 \times 17)$ scenarios for the SSES experiment. For each scenario, there were eight subjects who participated and each subject was exposed 20 times to the SSES experiment. In order to control the influences of the standard stimulus's location and time, a multilayer ABBA method is adopted to control the error in the experiment. Table 1 lists the specific control form of the multi-level ABBA method [13]. 
Table 1. Multi-level ABBA Technique.

\begin{tabular}{ccccc}
\hline $\begin{array}{c}\text { The order of displaying stimuli } \\
\text { (S-standard, C-control) }\end{array}$ & C-S & S-C & S-C & C-S \\
\hline Speed Change of comparison stimuli & Increase & Decrease & Decrease & Increase \\
\hline
\end{tabular}

According to the subjects' responses, the ratio that the control stimulus was perceived faster than the standard stimulus was graphed as y-axis and physical speed of standard stimulus was plotted as $\mathrm{x}$-axis. Using interpolation method, the 50\% percentile of the control stimulus speed was the SSES.

\section{Stimuli}

A standard stimulus is one of the combinations of speed $(60 \mathrm{~km} / \mathrm{h}, 90 \mathrm{~km} / \mathrm{h}$, and $120 \mathrm{~km} / \mathrm{h})$, design types (two bright, two dark, and four bright, four dark), SS (2 m and $4 \mathrm{~m}$ ), SOA (60 ms, $120 \mathrm{~ms}, 180 \mathrm{~ms}$, and $240 \mathrm{~ms}$ ). A control stimulus is one of the driving scenarios without grating under different speeds (from $40 \mathrm{~km} / \mathrm{h}-200 \mathrm{~km} / \mathrm{h}$, with intervals of $10 \mathrm{~km} / \mathrm{h}$ ). Figure 5 depicted one scenario of the standard stimulus and one scenario of the control stimulus, respectively.

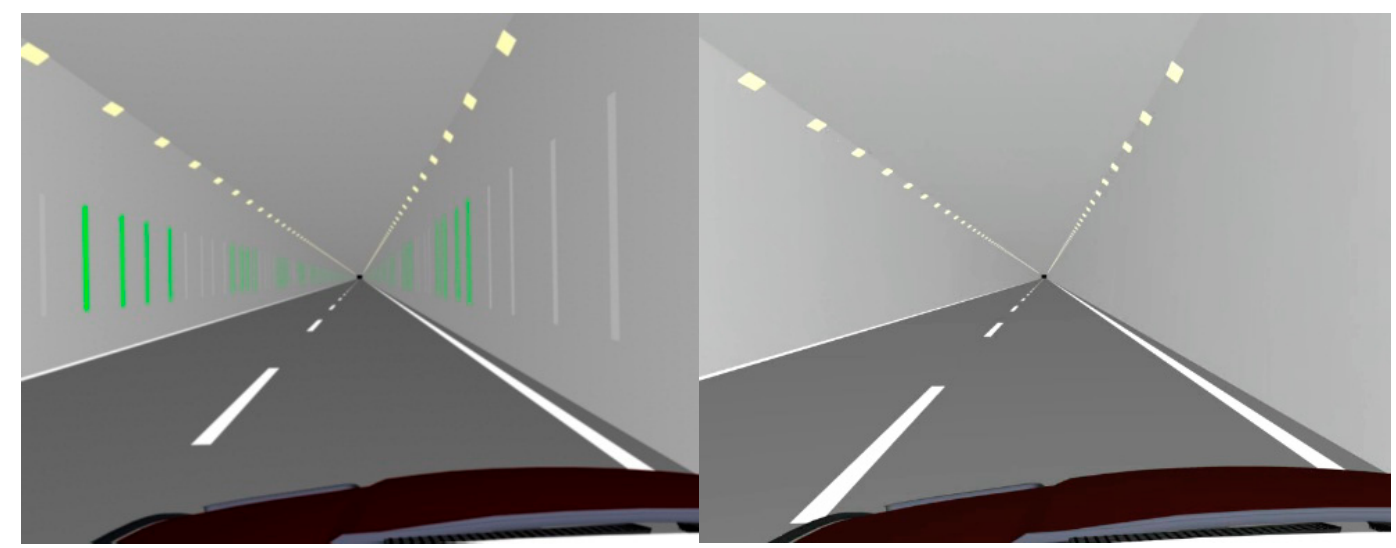

Figure 5. Scenarios of the standard stimulus and the control stimulus.

Results

The averages of SSES under different circumstances are obtained after summarizing the experiment result of the eight subjects, as shown in Figure 6:

From the results, we could obtain the following findings:

(1) SS and SOA have significant influence on the speed perception. When SS remains constant, higher speed can be perceived with the decrease in SOA, and when SOA remains constant, higher speed can be perceived with the decrease in SS.

When testing for statistical significance perception speed, the mean difference was significant at the 0.05 level using Analysis of Variance (ANOVA).

(2) When two bright, two dark or four bright, four dark gratings were used, participants will overestimate the speed. 


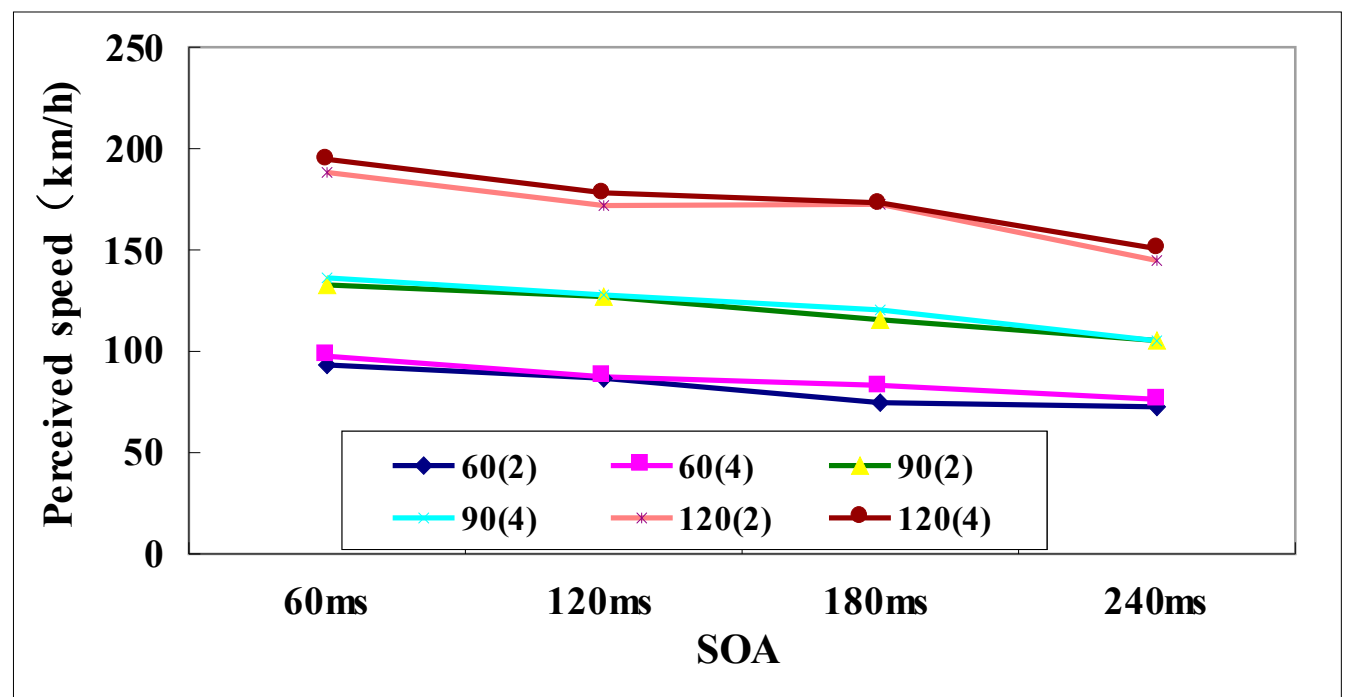

(a)

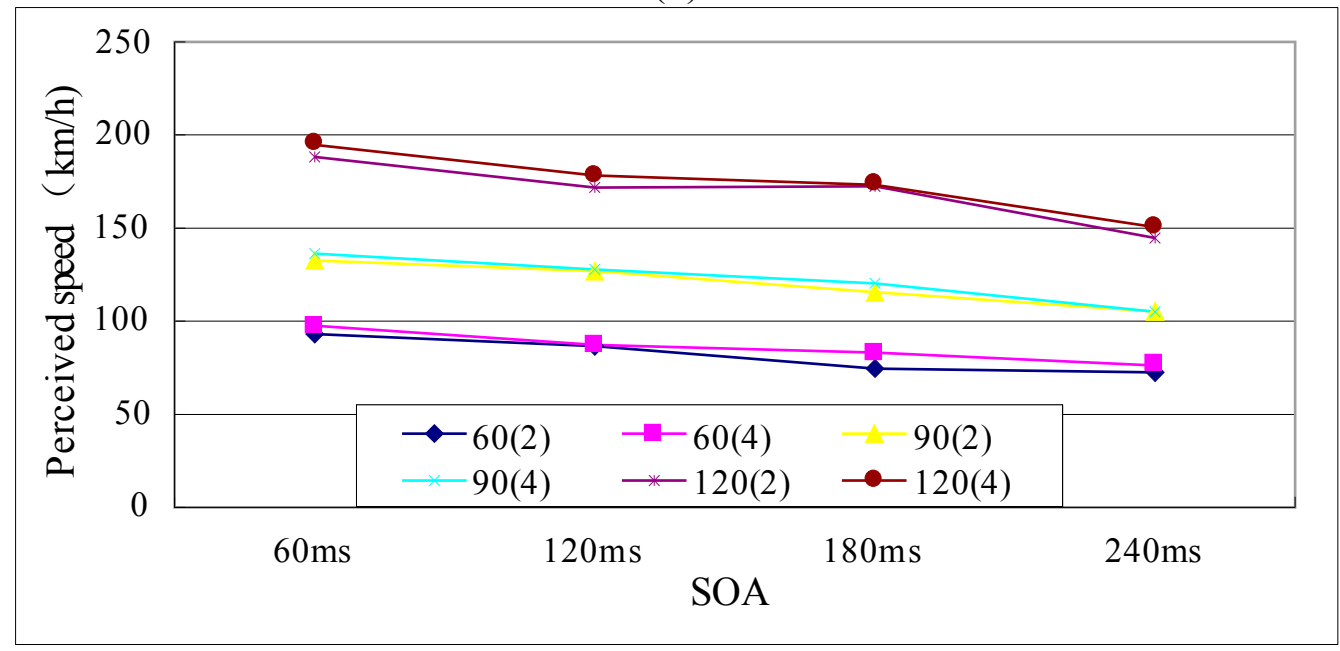

(b)

Figure 6. The relationship between the perceived speed, stimulus separation (SS), stimulus onset asynchrony (SOA) and physical speed. (a) Two bright two dark; (b) four bright four dark. 60 (2) means speed is $60 \mathrm{~km} / \mathrm{h}$, SS is $2 \mathrm{~m}$.

\section{Discussion and Conclusions}

\subsection{Optimal Form of Apparent Motion}

When one bright, one dark grating was used, no matter the value of SS and the SOA, participants tended to perceived grating as "flashing" or "unstable movement". The above result can be explained by the research work done by Anstis [14]. In his research, the impact of contrast on motion direction is studied. He adopted Michelson contrast [15] to approximate the contrast which was experienced in our experiments.

$$
\text { Michelson contrast }=\frac{\text { object luminance }- \text { background luminance }}{\text { object lum } \text { inance }+ \text { background luminance }}
$$

The states of two adjacent moments are shown in a and b, when grating with one bright, one dark form, at moment $\mathrm{t} 1$ (shown in Figure 7), stripe 1 and stripe 3 with higher contrast, as well as stripe 2 
with lower contrast. When $\mathrm{t} 1$ moment switches to $\mathrm{t} 2$ moment, observers may perceive that stripe 1 moves towards stripe 2 or stripe 3 moves towards stripe 2, and then motion direction is ambiguous. When grating with a two bright, two dark form, the states of two adjacent moments are shown in $\mathrm{c}$ and $\mathrm{d}$. At $\mathrm{t} 1$ moment, stripes 1 and stripe 2 have higher contrast, while stripes 3 and 4 have lower contrast. As stripes 1 and 2 have the same contrast, observers should consistently perceive stripe 2 as moving towards stripe 3. When grating with a four bright, four dark form, the perception condition is the same as with a two bright, two dark form.

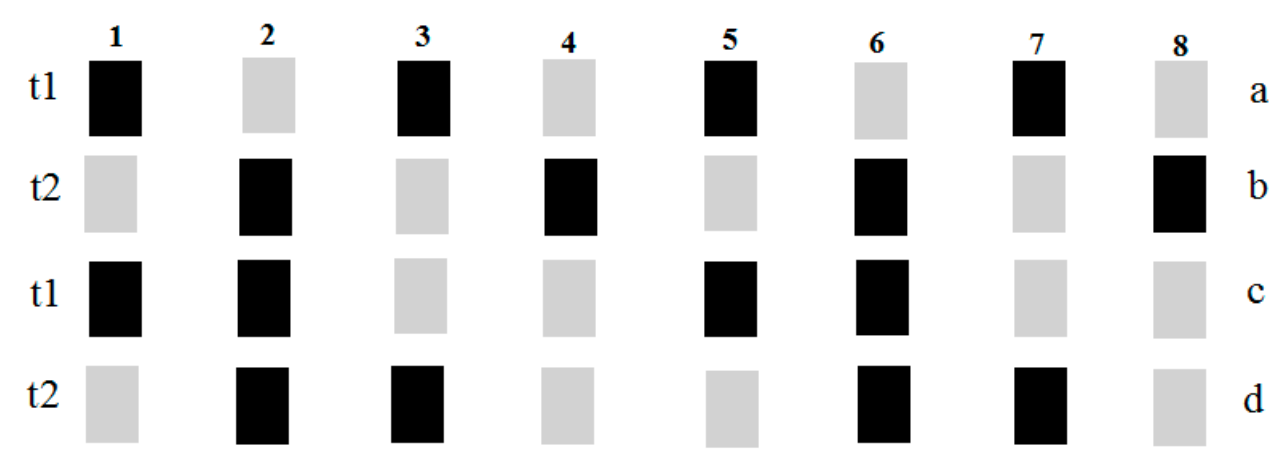

Figure 7. Layout of grating at adjacent moment.

\subsection{Driver's Speed Perception}

The speed (Va) of apparent motion can be determined from SS and SOA as Va $=$ SS/SOA. Table 2 shows the speed of apparent motion under circumstances of different SS and SOA. When SS is fixed, a lower SOA can lead to a greater Va. This is consistent with the finding that perceived speed increased as SOA decreased in experiment 2.

Table 2. Apparent Motion Speed with Different SS and SOA $(\mathrm{km} / \mathrm{h})$.

\begin{tabular}{ccc}
\hline SOA & $\mathbf{2 ~} \mathbf{~ m}$ & $\mathbf{4 ~ m}$ \\
\hline $60 \mathrm{~ms}$ & 120 & 240 \\
$120 \mathrm{~ms}$ & 60 & 120 \\
$180 \mathrm{~ms}$ & 40 & 80 \\
$240 \mathrm{~ms}$ & 30 & 60 \\
\hline
\end{tabular}

However, when SS varies, perceived speed cannot solely be considered with apparent speed. In addition, the impact of edge rate [16,17] should be taken into account. Edge rate is the amount of edge or intermittent that passed through observers' eyes in one unit time length. Once the distance between stripes is measured, edge rate will tell us the observer's speed. When edge rate (stripe grows thinner) increases, observer will perceive higher speed. Referring to the definitions of edge rate, there is a trade-off relationship with apparent motion speed. When SOA remains constant, Va increases as SS increases, while ER decreases as SS increases. In experiment 2, with SOA remaining constant, the perceived speed was slightly higher when SS equaled $4 \mathrm{~m}$ than when SS was $2 \mathrm{~m}$. To some extent, this phenomenon demonstrated that, in the environment of our experiment, apparent motion speed may have had a 
slightly greater impact on perceived speed than edge rate, but this assumption needs to be verified in the future research.

\subsection{Optimal Form for Speed Control}

According to the results of experiments, the best parameters of apparent motion grating for speed reduction are: two bright, two dark form, SS as $4 \mathrm{~m}$ and SOA as $60 \mathrm{~ms}$. Under these conditions, the average perceived speed of eight observers reaches the maximum; meanwhile, the standard deviation is lower than that of the four dark, four bright case.

\section{Acknowledgments}

This work was supported by the National Natural Science Foundation of China (NO.50778299) and the Scientific and Technological Project of the Ministry of Transport of the People's Republic of China (NO.2010353342240). We gratefully acknowledge the assistance of experiment participants.

\section{Author Contributions}

Bing Liu and Shunying Zhu designed research; Hong Wang and Jing Xia performed research and analyzed the data; Bing Liu wrote the paper. All authors read and approved the final manuscript.

\section{Conflicts of Interest}

There is no conflict of interest regarding the publication of this article.

\section{References}

1. Ministry of Public Security of the People's Republic of China. White Paper on Road Traffic Accident in China; China Communications Press: Beijing, China, 2011.

2. Theeuwes, J. Self-Explaining Roads: An exploratory study. In Transportation Research Record: Journal of the Transportation Research Board; Transportation Research Board of the National Academies: Washington, DC, USA, 1994.

3. Gepshtein, S.; Kubovy, M. The Lawful Perception of Apparent Motion. J. Vision 2007, 7, 1-15.

4. Recarte, M.A.; Nunes, L.M. Perception of Speed in an Automobile: Estimation and Production. J. Exp. Psychol. Appl. 1996, 2, 291-304.

5. Wertheimer, M. Experimentelle Studien uber dasSehen von Bewegung. Zeitschrift für Psychologie 1912, 61, 161-265.

6. Hock, H.S.; Gilroy, L.A.; Harnett, G. Counter-changing Luminance: A Non-fourier, Non-attentional Basis for the Perception of Single-element Apparent Motion. J. Exp. Psychol. Hum. Perce. Perform. 2002, 28, 93-112.

7. Hock, H.S.; Kogan, K.; Espinoza, J.K. Dynamic State-dependent Thresholds for the Perception of Single-element Apparent Motion: Bistability from Local Cooperativity. Perce. Psych. 1997, 59, 1077-1088.

8. Zhuo, Y.; Zhou, T.G.; Rao, H.Y.; Wang, J.J.; Meng, M.; Chen, M.; Zhou, C.; Chen, L. Contributions of the Visual Ventral Pathway to Long-range Apparent Motion. Science 2003, 299, 417-420. 
9. Tsuda, R.; Ueno, S. Perception of Visual Apparent Movement in Central and Peripheral Vision. IEEE 1993, doi:10.1109/IEMBS.1993.978417.

10. Yamamoto, K.; Uchida, K.; Sagawa, Y.; Tanaka, T.; Sugie, N. Quantitative Measurements of Driver's Motion After-Effect. Intell. Vehicle Symp. 2002, 2, 336-343.

11. Shen, H.; Shimodaira, Y.; Ohashi, O. Speed-Tuned Mechanism and Speed Perception in Human Vision. Syst. Comput. Japan 2005, 36, 1718-1727.

12. Jäkel, F.; Wichmann, F.A. Spatial Four-alternative Forced-choice Method is the Preferred Psychophysical Method for Naïve Observers. J. Vision 2006, 6, 1307-1322.

13. Ruud, G.J.; Meulenbroeka Galena, G.P.; van Hulstijnb, M.; Hulstijna, W.; Bloemsaat, G. Muscular Co-contraction Covaries with Task Load to Control the Flow of Motion in Fine Motor Tasks. Biol. Psychol. 2005, 68, 331-352.

14. Anstis, S. Moving Objects Appear to Slow Down at Low Contrasts. Neural Netw. 2003, 16, 933-938.

15. Michelson, A.A. Studies in Optics; Dover Publication, Inc.: New York, NY, USA, 1995.

16. Wickens, C.D.; Hollands, J. Engineering Psychology and Human Performance; Prentice Hall, Inc.: Upper Saddle River, NJ, USA, 1999.

17. Denton, G.G. The Influence of Visual Pattern on Perceived Speed. Perception 1980, 9, 393-402.

(C) 2015 by the authors; licensee MDPI, Basel, Switzerland. This article is an open access article distributed under the terms and conditions of the Creative Commons Attribution license (http://creativecommons.org/licenses/by/4.0/). 